\title{
Peptide Receptors - From Selective Molecular Recognition Towards Catalytic Activity
}

\author{
Helma Wennemers*
}

\begin{abstract}
The development of diketopiperazine receptors, a class of two-armed receptors that bind peptides with high selectivity, has been achieved by a combination of on-bead combinatorial binding assays and conformational analysis using X-ray crystal structure analysis and NMR spectroscopy. Furthermore, a general method for the discovery of catalysts within split-and-mix libraries has been developed that is based on the co-immobilization of the potential catalyst and one reactant on the same bead.
\end{abstract}

Keywords: Catalysis · Combinatorial chemistry · Molecular recognition · Peptide receptors . Split-and-mix libraries

\section{Introduction}

Substrate-specific catalytic transformations require the selective binding of the catalyst to the substrate. The understanding of the principles that govern molecular recognition and the ability to create specific molecular hosts is therefore fundamental to the design of new catalysts. Nature uses amino acids with their repertoire of functional groups as basic components of enzymes with excellent catalytic performances that have evolved over many million years. Combinatorial chemistry can be regarded as the chemist's attempt to mimic the natural evolution principles of random mutations and selection for the fittest [1]. It therefore provides a good tool to test whether peptides with lower molecular weights than natural enzymes can also act as selective catalysts. To approach this goal peptidic molecules with good binding properties are necessary, as well as screening methodologies that allow not only

\footnotetext{
${ }^{*}$ Correspondence: Dr. H. Wennemers Department of Chemistry University of Basel St. Johanns-Ring 19 $\mathrm{CH}-4056$ Basel

Tel.: +41612671146

Fax: +41612671105

E-Mail: Helma.Wennemers@unibas.ch
}

screening for selective binding but also for catalytic activity.

Screening for selective binders among the members of a split-and-mix library is easily accomplished by mixing a receptor that is labeled by e.g. a dye, a fluorophore or radioactivity with the library of potential guests [2]. The labeled receptor will concentrate on those beads that carry molecules with affinity for the receptor which can be easily identified with a microscope. Using such on-bead screenings of splitand-mix libraries we have developed diketopiperazine receptors, a class of two-armed receptors with good binding properties towards peptides [3-5].

Screening of split-and-mix libraries for catalytic activity is a more challenging task since the catalyst on a single bead is - naturally - unchanged by a catalytic reaction and reactants as well as products are free to diffuse in the surrounding solution. As a result, most methods have been established for rather specific reactions and rely on the formation of either colored products or on a $\mathrm{pH}$-change during the reaction [6-9]. We have developed a general method for the discovery of catalysts in split-and-mix libraries that allows for the testing of catalysts for principally any bimolecular reaction [10].

The following account will summarize both the development of diketopiperazine receptors and the combinatorial method for the discovery of catalysts.

\section{Selective Peptide Recognition by Diketopiperazine Receptors}

Besides applications in the development of catalysts, the increasing need for therapeutics and sensors asks for the development of synthetic receptors that recognize small peptides selectively. Initially, we therefore developed a class of receptors with members capable of principally recognizing any desired peptide selectively. Such a class of receptors should contain a rigid backbone, functional groups and enough moieties that can be varied easily. We envisioned that the two-armed diketopiperazine receptors shown in Fig. 1 fulfill all of these requirements [3]: The central diketopiperazine based on $\gamma$-amino-proline should function as a rigid structure-directing back-

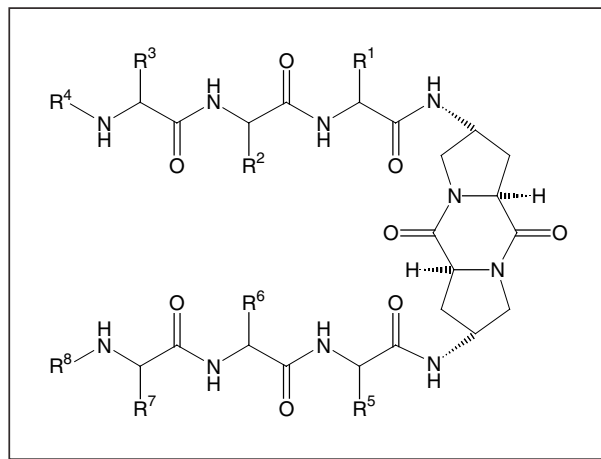

Fig. 1. General structure of diketopiperazine receptors 
bone and as an anchor for two tripeptidic side chains, the receptor arms. These allow the introduction of functional groups for the formation of non-covalent interactions such as hydrogen bonds, ionic and hydrophobic interactions. Furthermore, the receptor structure offers opportunities for structural and functional variations and is accessible by a simple synthesis both in solution and on solid supports.

\subsection{Combinatorial Binding Studies} receptors are indeed able to bind to peptides we prepared receptor prototypes and tested them for their binding properties towards a tripeptide library [3]. Our choice fell on the five receptor prototypes 1-5 with similar yet distinctly different structures: The two arms of each receptor were identical and consisted of dye-marked L- or D-tyrosines as initial amino acids followed by L-phenylalanine and a combination of either L-asparagine or L-glutamine (Fig. 2). The red azo-dye Disperse Red was used as an inert marker to visualize binding of the receptors to the peptide library.

To test the binding properties of the receptor prototypes a tripeptide library with the general structure Ac-AA3-AA2-AA1$\mathrm{NH}\left(\mathrm{CH}_{2}\right)_{5} \mathrm{CONH}-\mathrm{PS}$ (PS = Polystyrene Resin) was prepared by the protocol for encoded [11] split synthesis [12]. The library contained maximally $29^{3}=24389$ different acetylated tripeptides, since 29 different D- and L-amino acids were employed in each position. When the library was mixed and equilibrated with dilute solutions of the receptor prototypes in chloroform solution,
To examine whether diketopiperazine

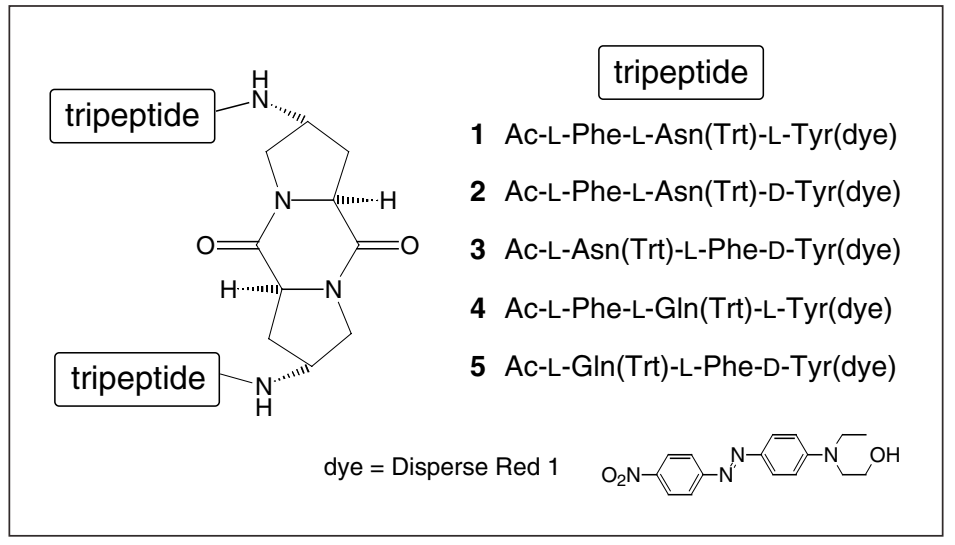

Fig. 2. Diketopiperazine receptor prototypes only a few beads picked up the red color of the receptors (Fig. 3). Particularly high selectivities were observed in the assays of receptors $\mathbf{1}$ and $\mathbf{5}$ where only approximately one bead out of 5000 turned red.

Isolation of the red beads followed by analysis of the peptide sequences on these

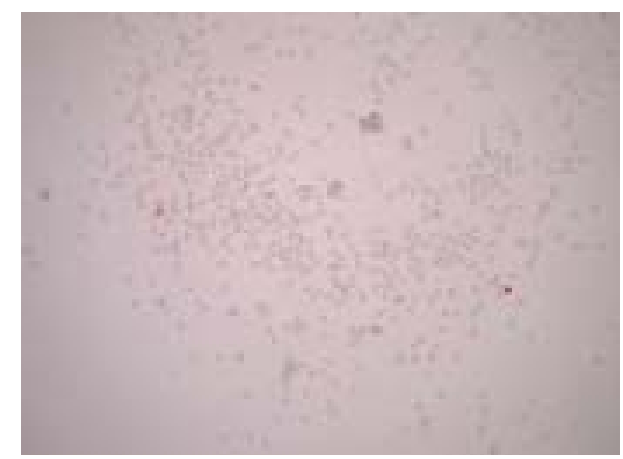

Fig. 3. Microscope view of the on-bead assay of receptor 1 with the tripeptide library Ac-AA3AA2-AA1- $\mathrm{NH}\left(\mathrm{CH}_{2}\right)_{5} \mathrm{CONH}-\mathrm{PS}$
Table. Binding specificities of the receptor prototypes 1-5 for tripeptides within the library Ac-AA3-AA2-AA1- $\mathrm{NH}\left(\mathrm{CH}_{2}\right)_{5} \mathrm{CONH}-\mathrm{PS}$

\begin{tabular}{llllll} 
& AA3 & AA2 & AA1 & freq. found [\%] & freq. expec. [\%] \\
\hline & D-Val/D-Ala & D-Hph & D-His & 100 & 0.04 \\
2 & D-Ala/D-Val & L-Asn/L-Gln & L-Ala/Gly & 43 & 0.03 \\
& L-Asn & D-Pro & L-Hph & 57 & 0.02 \\
3 & D-Ala/D-Val & L-Hph & L-Ser/L-Thr & 46 & 0.08 \\
& D-Hph & L-Ala & L-Gln/L-Asn & 23 & 0.04 \\
& D-Hph & L-Hph & L-Ala & 17 & 0.08 \\
4 & D-Ala/D-Val & D-Hph & D-His & 34 & 0.04 \\
& L-Ala/L-Leu & L-Gln & D-Hph & 37 & 0.04 \\
& D-Gln & D-Hph & D-Val/D-Leu & 20 & 0.04 \\
5 & D-Ala/D-Val/D-Leu & L-Hph & L-Asn & 100 & 0.06
\end{tabular}

aThe 'frequency found' column lists the percentage of beads selected in the receptor binding assay for the indicated peptide sequence. The 'frequency expected' column lists the expected frequency for the particular tripeptide sequence if the beads were picked randomly. The comparison between the percentage of 'frequency found' and 'frequency expected' is a measure for the selectivity level of the receptor.

bHph = hydrophobic amino acid can be either Gly, Ala, Val, Leu or Phe. beads revealed remarkable binding selectivities: Each of the five structurally similar receptor prototypes is selective for different tripeptides within the library (Table). For example, receptor 1 selects preferentially for peptides with a D-histidine followed by two hydrophobic D-amino acids, while receptor $\mathbf{5}$ prefers peptides with an L-asparagine following a pair of hydrophobic L- and D-amino acids [3]. These binding results demonstrate that small modifications in the receptor structure induce significant changes in the binding preferences. Equally selective and structurally responsive binding selectivities have also been observed when the binding properties of the receptors were examined towards the same tripeptide library but with the sidechain protecting groups still attached [4].

\subsection{Binding Affinity Measurements}

Since solubility problems hampered the elucidation of the binding modes and strengths of the observed intermolecular interactions in solution phase, we resorted to a solid-phase binding assay for the determination of the binding affinities between the receptors and their preferred peptide. This assay provides a convenient estimate of the intermolecular binding strength simply by measuring the UV/Vis absorbance of the colored receptor before and after the incubation with the solid-supported peptide [13]. Using a polystyrene support with a loading of $0.11 \mathrm{mmol} \mathrm{g}^{-1}$ receptor 1 binds the selected peptide Ac-D-Val-D-Val-D-Hislinker-PS with an affinity of $\Delta \mathrm{G}=-5.7 \mathrm{kcal}$ $\mathrm{mol}^{-1}$. The binding strengths of receptor 4 that had a less pronounced selectivity for this peptide is only $\Delta \mathrm{G}=-4.8 \mathrm{kcal} \mathrm{mol}^{-1}$ [13]. Changes in the structure of the peptide led to significantly decreased binding affinities of $\leq 1.5 \mathrm{kcal} \mathrm{mol}^{-1}$ indicating that the binding selectivities of the combinatorial assay correlate well with the binding affinities [3]. 


\subsection{Structural Prerequisites and Conformational Analysis}

What are the reasons for the observed highly selective binding properties? To gain deeper understanding of the structural prerequisites of two-armed diketopiperazine receptors we studied the binding properties of truncated receptors and two-armed molecules where the central diketopiperazine was exchanged against other diamines.

Receptor fragments containing only two amino acids in the side arms or a single arm only, did not show any binding to the members of the tripeptide library. Thus, a receptor structure with two tripeptidic arms is the minimal structure necessary to observe peptide binding [3].

Equally or even more important is the choice of the template [5]: Two-armed molecules based on diamines such as $(1 R, 2 R)$ diaminocyclohexane, 1,3-di(aminomethyl) cyclohexane, 1,7-diaminoheptane or 3,7diamino-1,5-diazabicyclo[3.3.0]octane do not interact with any of the $\approx 24000$ peptides within the tripeptide library which indicates that the diketopiperazine derived from trans- $\gamma$-aminoproline [14] is an excellent template for two-armed receptors. Most remarkably, even receptors built upon the diketopiperazine derived from $c i s$ - $\gamma$-aminoproline [14] showed worse binding selectivities (Fig. 4). The binding properties of these "cis-diketopiperazine" receptors proved highly dependent on the configuration of the amino acid closest to the template.

To understand why the diketopiperazine derived from trans- $\gamma$-aminoproline ('transdiketopiperazine') provides a superior structural basis for two-armed receptors compared to particularly the diastereomeric cis-diketopiperazine, we analyzed the conformation of diketopiperazine derivatives in the solid state by X-ray single crystal structure analysis as well as by NMR studies in solution [5]. For these studies we used the bis-acetylated cis- and trans-diketopiperazines $\mathbf{6}$ and $\mathbf{7}$ as minimal fragments of the receptors since it was not possible to obtain crystals of the two-armed receptors that were suitable for X-ray structure analysis and since the signals in the NMR spectra of the receptors largely overlap.

The major difference between the conformation of $\mathbf{6}$ and $\mathbf{7}$ is the orientation of the $\mathrm{N}$-acetyl groups. In the crystal structure of the trans-diketopiperazine $\mathbf{6}$, the $\mathrm{N}$-acetyl groups occupy the pseudo-axial positions at the $\mathrm{C} \gamma$-atoms, in the cis-diketopiperazine 7 the pseudo-equatorial positions (Fig. 5). As a result, the conformation of the trans-diketopiperazine resembles a turn while the cis-diketopiperazine is a rather linear structural element in the solid state.

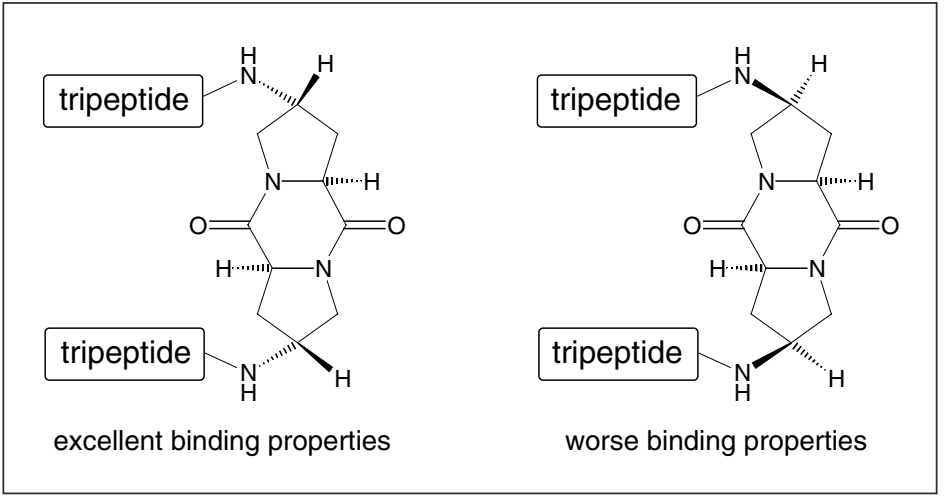

Fig. 4. Trans- and cis-diketopiperazine receptors [14]

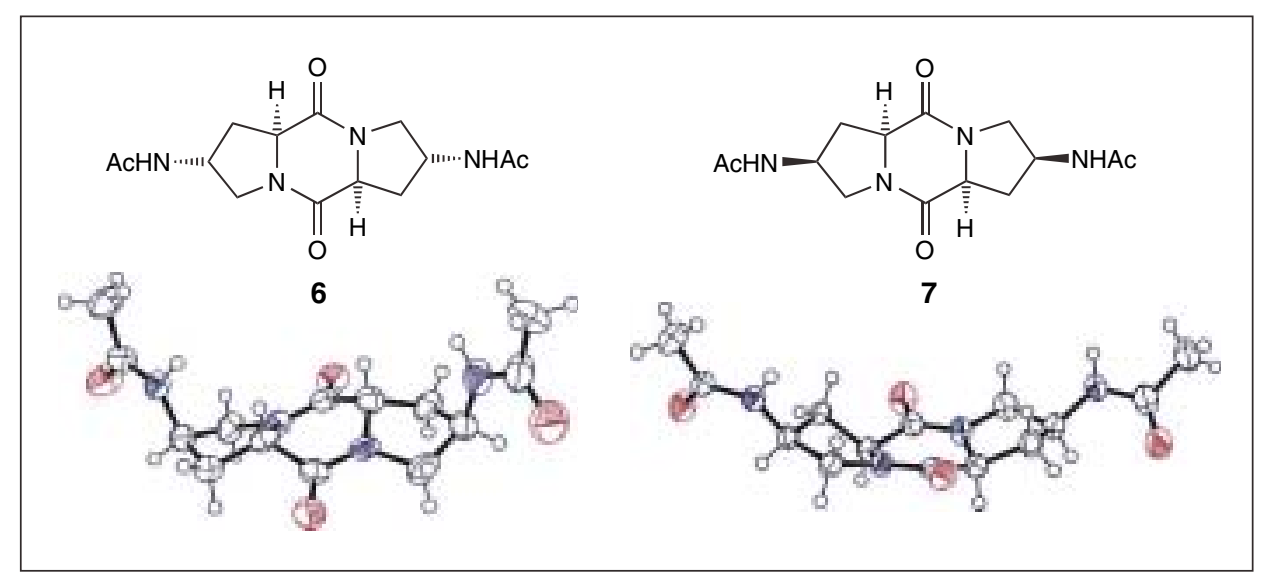

Fig. 5. Crystal structures of the trans-diketopiperazine 6 and cis-diketopiperazine 7

NMR spectroscopic studies revealed that the turn conformation of the transdiketopiperazine is also the major conformation occurring in solution. In contrast, the cis-diketopiperazine exists in chloroform solution in a fast approximately $1: 1$ equilibrium between conformations with the $\mathrm{N}$-acetyl groups in pseudo-equatorial positions and pseudo-axial positions. Thus, the conformation of the cis-diketopiperazine is very flexible while the trans-diketopiperazine is a conformationally rigid molecule with a well-defined turn. In combination with the combinatorial binding studies with two-armed molecules built on other rigid diamines, the conformational analysis revealed that this specific turn geometry provides an ideal base for twoarmed molecules with excellent binding properties towards short peptides.

Based on these initial results we have prepared a library of diketopiperazine receptors and are currently testing their binding properties towards dye-marked peptides not only in organic solvents but also in aqueous media. Furthermore, diketopiperazine receptors are being tested in the development of sensors and in the area of material sciences.

\section{Discovery of Catalysts in Split-and-Mix Libraries}

Compared to the many applications for studies in molecular recognition, the use of combinatorial chemistry for catalyst development is still in its infancy. However, particularly for the development of peptidic catalysts and ligands where rational design is hampered by the many degrees of freedom of even simple di- and tripeptides, combinatorial chemistry holds the potential to become an important tool. In contrast to the testing of split-and-mix libraries for selective binding partners, the detection of catalytically active members represents a greater challenge: A catalytically active library member is - naturally - left unchanged in the course of the reaction and reactants and products can freely diffuse to and away from the bead. This intrinsic challenge has to be met by smart screening methodologies. Successful methods rely on the use of IR-thermography [6], fluorescent $\mathrm{pH}$-sensors [7], insoluble colored reaction products [8] or the use of gels as reaction media [9]. However, except for IR-thermography these methods have been developed for specific reactions and rely either 
on the formation of colored products or a $\mathrm{pH}$ change during the reaction. Thus, we have developed a method that can be applied to the search for catalysts for most bimolecular reactions [10].

Our concept for the visualization of catalysts among the members of a split-andmix library is based on the immobilization of one reaction partner along with each library member, the potential catalyst, on the same bead. This can be easily accomplished by a bifunctional linker that carries on one end the reactant $\mathbf{A}$ and on the other end the library member. If the reaction partner $\mathbf{B}$ is labeled with e.g. a dye, a fluorophor, or radioactivity a reaction between $\mathbf{A}$ and $\mathbf{B}$ leads to the covalent attachment of the marker to those beads that carry catalytically active library members (Fig. 6). These beads can then easily be detected with a microscope.

We evaluated the feasibility of the method by testing the members of a tripeptide library for their ability to catalyze the acylation of an alcohol (reactant $\mathbf{A}$ ) by a dye-marked pentafluorophenyl (Pfp) ester (reactant B). The library was designed not to contain amino acids that could be selfacylated, thus, Ser, Thr, Lys and Cys were not part of the library. His was incorporated as a known weak acylation catalyst and was expected to be selected. After reacting the library with the dye-marked Pfp-ester and thorough washings, several beads remained red indicating the covalent attachment of the dye to the resin beads. Analysis of the peptides on these red beads showed that each active bead carried at least one His.

This method should be applicable to the search for catalysts for principally any bimolecular reaction where one reaction partner can be attached to a solid support and the other labeled with a dye, a fluorophore or radioactivity.

\section{Outlook}

Amino acids and small peptides are not only excellent chiral ligands for metal based catalysts but have the potential to serve as catalysts for several organic reactions [15]. Advantages such as facile preparation, chirality, functional and structural diversity to fine-tune for a desired substrate, render peptidic catalysts ideal candidates for substrate specific transformations. Our combinatorial assay for the discovery of catalyst should therefore provide an excellent tool to evaluate libraries of peptides and particularly of diketopiperazine receptors as catalysts for bimolecular reactions such as aldol reactions and cycloadditions.

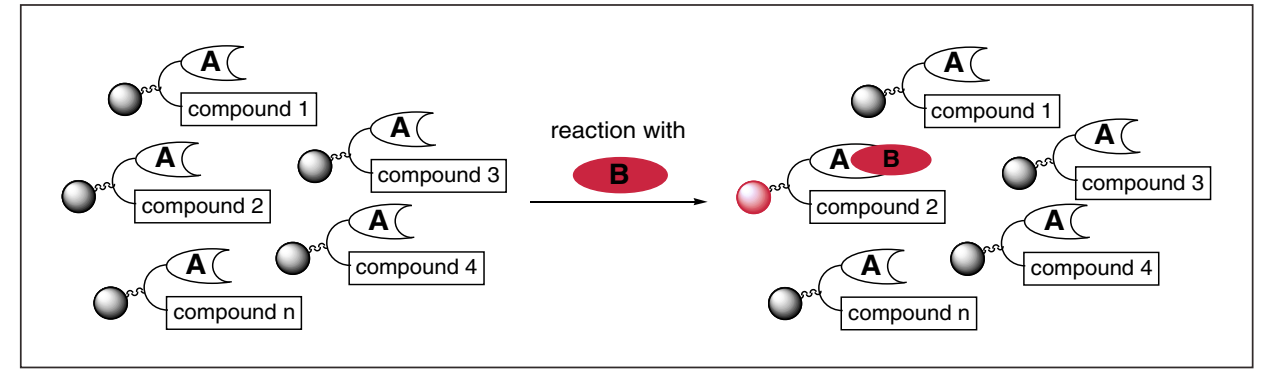

Fig. 6. General concept for the discovery of catalysts in one-bead-one-compound libraries. In the example only compound $\mathbf{2}$ is an active catalyst.

\section{Acknowledgements}

I am most grateful to my $\mathrm{PhD}$ students Matteo Conza, Jessica Grun, Philipp Krattiger, Matthias Nold, and Louis-Sebastian Sonntag for their enthusiasm and endurance in carrying out the work. I thank the Swiss National Science Foundation for financial support and in particular the BACHEM company for the Bachem assistant professorship.

Received: March 21, 2003

[1] K.C. Nicolaou, R. Hanko, W. Hartwig, 'Handbook of Combinatorial Chemistry', Wiley VCH, 2002.

[2] W.C. Still, Acc. Chem. Res. 1996, 29, 155.

[3] H. Wennemers, M. Conza, M. Nold, P. Krattiger, Chem. Eur. J. 2001, 7, 3342.

[4] M. Conza, H. Wennemers J. Org. Chem. 2002, 67, 2696.

[5] H. Wennemers, M.C. Nold, M.M. Conza, K.J. Kulicke, M. Neuburger, Chem. Eur. J. 2003, 9, 442.

[6] S.J. Taylor, J.P. Morken, Science 1998, 280, 267.

[7] A. Berkessel, D.A. Hérault, Angew. Chem. Int. Ed. 1999, 38, 102.

[8] a) G.T. Copeland, S.J. Miller, J. Am. Chem. Soc. 2001, 123, 6496; b) G.T. Copeland, S.J. Miller, J. Am. Chem. Soc. 1999, 121, 4306.

[9] a) M. Müller, T.W. Mathers, A.P. Davis, Angew. Chem. Int. Ed. 2001, 40, 3813; b) R.F. Harris, A.J. Nation, G.T. Copeland, S.J. Miller, J. Am. Chem. Soc. 2000, 122, 11270.

[10] P. Krattiger, C. McCarthy, A. Pfaltz, H. Wennemers, Angew. Chem. Int. Ed. 2003, 42, 1722-1724.

[11] M.H.J. Ohlmeyer, R.N. Swanson, L.W. Dillard, J.C. Reader, G. Asouline, R. Kobayashi, M.H. Wigler, W.C. Still, Proc. Natl. Acad. Sci. USA 1993, 90, 10922.

[12] a) Á. Furka, F. Sebestyén, M. Asgedom, G. Dibô, Int. J. Pept. Protein Res. 1991, 37, 487; b) K.S. Lam, S.E. Salmon, E.M. Hersh, V.J. Hruby, W.M. Kazmierski, R.J. Knapp, Nature, 1991, 354, 82.

[13] M. Conza, H. Wennemers, Chem. Commun. 2003, 866.

[14] 'trans' and 'cis' refer to the relative configuration of the substituents at $\mathrm{C} \alpha$ and $\mathrm{C} \gamma$ of each proline.

[15] For a review see E.J. Jarvo, S. Miller, Tetrahedron 2002, 58, 248. 